Lösungsmittels im Vakuum wurde der ölige Rückstand durch Erhitzen in Ligroin gelöst und filtriert. Bereits nach kurzem Stehen kristallisierte das Azin (9,7 g) in Form feiner gelber Nadeln vom Schmp. $127-129^{\circ}$ aus. Dieses Präparat ergab mit dem oben erhaltenen keine Schmelzpunktsdepression.

Das Ketazin ließ sich durch mehrstdg. Kochen in Methanol und halbkonz. $\mathrm{HCl}$ unter $\mathrm{N}_{2}$-Abgabe zur Acetylverbindung verseifen, die durch Wasserdampfdestillation aus dem Reaktionsmedium isoliert werden konnte.

Phenyl-[methyl- (x-methyl-cyclopentadienyl) - mangan-tricarbonyl]. a zimeth y len

(Tab. 1, lfd. Nr. 10)

$2,7 \mathrm{~g} \quad(0,01 \quad \mathrm{Mol}) \quad$ [(x-Acetyl-1-methyl-cyclopentadienyl)-mangan-tricarbonyl]-hydrazon wurden zusammen mit $1,1 \mathrm{~g}(0,01 \mathrm{Mol})$ Benzaldehyd 5 Stdn. unter Stickstoff in einem Ölbad auf $135^{\circ}$ erhitzt. Danach wurde das Umsetzungsprodukt in der Siedehitze in Ligroin gelöst und filtriert. Nach mehrstdg. Aufbewahren im Kühlschrank kristallisierte das "gemischte“ Ketazin und schmolz nach weiterem Umkristallisieren bei $87-88^{\circ}$, gelbe prismenartige Kristalle $(2,2 \mathrm{~g})$.

Die übrigen in Tab. 1 aufgeführten Verbindungen wurden nach analogen Vorschriften hergestellt.

\section{$N, N-\mathrm{B}$ is $-\{(\mathrm{m}$ e thy $\mathrm{l}-[(\mathrm{x}-\mathrm{m}$ e thy $\mathrm{l}-\mathrm{c}$ y c lo - pentadienyl) - mangan-tricarbonyl)]. methylen - imidyl-succin a mid}

(Tab. 1, lfd. Nr. 17)

$4,1 \mathrm{~g}(0,015 \mathrm{Mol})$ des durch Umsetzung von $\mathrm{x}$-Acetyl-II mit Hydrazin erhaltenen Hydrazons und $1,9 \mathrm{~g}$ $(0,019 \mathrm{Mol})$ Triäthylamin wurden in $100 \mathrm{~cm}^{3}$ Äther gelöst und tropfenweise unter Kühlung und Rühren mit
1,24 g (0,008 Mol) Bernsteinsäuredichlorid versetzt, wobei sich unter spontaner Erwärmung sofort ein gelblicher Niederschlag bildete. Es wurde dann noch eine Stde. unter Rühren am Rückfluß gekocht und nach dem Abkühlen im Scheidetrichter mit $100 \mathrm{~cm}^{3}$ Wasser durchgeschüttelt. Die gelbe ätherische Phase wurde abgetrennt und die wäßrige Schicht mehrmals ausgeäthert. Die Ätherlösung wurde zunächst mit verd. Schwefelsäure, dann mit Wasser bis zur neutralen Reaktion gewaschen und der Äther nach dem Trocknen mit $\mathrm{Na}_{2} \mathrm{SO}_{4}$ abdestilliert. Der braune, zähflüssige Rückstand wurde mit Chloroform versetzt und nunmehr bis zur völligen Kristallisation an der Wasserstrahlpumpe belassen. 4,0 g vom Schmp. $215-220^{\circ}$. Nach 2-maligem Umkristallisieren aus Propanol lag der Schmp. bei 231 bis $233^{\circ}$ : gelbe Kristalle, die in Ligroin sehr schwer, in Methanol und Äthanol schwer löslich sind; leicht löslich in Aceton, Tetrachlorkohlenstoff und Chloroform.
$[(\mathrm{x}-\mathrm{Acet} \mathrm{y} \mathrm{l}-1-\mathrm{meth} \mathrm{y} \mathrm{l}-\mathrm{c}$ yclopentadieny $\mathrm{l})$ - mangan-tricarbonyl]- $N$ - benzyl- hydrazon

(Tab. 1, lfd. Nr. 18)

4,1 g $(0,015 \mathrm{Mol})$ [(x-Acetyl-1-methyl-cyclopentadienyl)-mangan-tricarbonyl]-hydrazon, gelöst in $60 \mathrm{~cm}^{3}$ Ligroin, wurden mit $3,55 \mathrm{~g}(0,035 \mathrm{Mol})$ Triäthylamin sowie $4.3 \mathrm{~g}(0,034 \mathrm{Mol})$ Benzylchlorid versetzt und 16 Stdn. am Rückfluß zum Sieden erhitzt. Nach dem Erkalten wurde von dem ausgefallenen farblosen Niederschlag abgesaugt, dieser mit Äther gewaschen und die vereinigten Filtrate im Vakuum bis zum Öl eingeengt. Danach wurde unter Stickstoff im Hochvakuum destilliert. Es wurden $1,9 \mathrm{~g}$ eines orangegelben Öles vom Sdp. $111-129^{\circ} / 0,8$ Torr erhalten, das bei nochmaliger Destillation bei $119-121^{\circ}$ unter einem Druck von 0,7 Torr siedete, $\mathrm{n}_{\mathrm{D}}^{20}: 1,5900$.

\title{
Sterische Zuordnung der Prephensäure
}

\author{
Von H. Plieninger und G. Keilich \\ Aus dem Organisch-chemischen Institut der Universität Heidelberg \\ (Z. Naturforschg. 16 b, 81-84 [1961] ; eingegangen am 6. Oktober 1960)
}

\begin{abstract}
Ba-Prephenat wird in Gegenwart von Palladium/Bariumsulfat hydriert. Beim papierchromatographischen Vergleich des 2.4-Dinitrophenylhydrazons des Reduktionsprodukts mit den Dinitrophenylhydrazonen der beiden stereoisomeren synthetischen Tetrahydroprephensäuren III und IV zeigte sich Identität mit III. Daraus folgt Struktur X für Prephensäure.
\end{abstract}

Die Prephensäure (I) nimmt eine zentrale Stellung bei der Biosynthese aromatischer Aminosäuren ein ${ }^{1-4}$. Sehr wahrscheinlich ist sie ebenfalls eine

1 B. D. Davis, Science [Washington] 118, 251 [1953].

2 M. Katagiri u. R. Sato, Science [Washington] 118, 250 [1953].

3 U. Weiss, C. Gilvarg, E. S. Mingioli u. B. D. Davis, Science [Washington] 119, 774 [1954].

- B. D. Davis, A Symposium on Amino Acid Metabolism.
Zwischenstufe bei der Biosynthese des Cumarins ${ }^{5}$ und des Lignins ${ }^{6}$. Ihre Bedeutung als Vorläufer zahlreicher Alkaloide wird diskutiert ${ }^{7}$.

W. D. McElroy and H. S. Glass, Editors, John Hopkins Press, Baltimore 1955, S. 799.

5 F. WeYGand u. H. Wend, Z. Naturforschg. 14b, 421 [1959].

6 K. Kratzl u. H. Faigle, Z. Naturforschg. 15 b, 4 [1960].

7 E. Wenkert, Experientia [Basel] XV, 165 [1959]. 

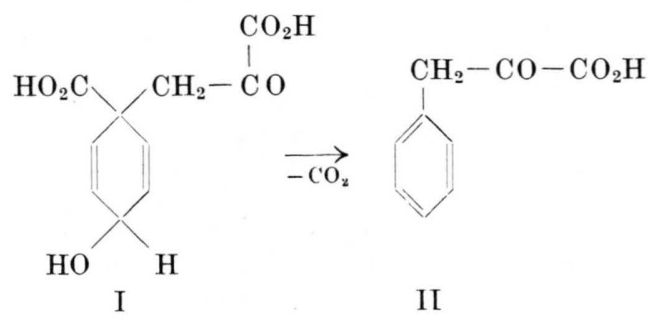

Die Aufstellung der Strukturformel I durch Weiss, Gilvarg, Mingioli und Davis ${ }^{3}$ ergab sich aus folgendem: Die Analyse des Bariumsalzes spricht für eine Dicarbonsäure der Formel $\mathrm{C}_{10} \mathrm{H}_{8} \mathrm{O}_{6} \mathrm{Ba} \cdot \mathrm{H}_{2} \mathrm{O}$. Nach Reduktion der Ketogruppe ist bei $260 \mathrm{~m} \mu$ keine Absorption vorhanden, so daß eine aromatische Struktur ausscheidet. Bei Gegenwart von Platin werden $3-4$ Mole Wasserstoff aufgenommen. In saurem Medium erfolgt schneller Übergang in Phenylbrenztraubensäure (II) unter Entwicklung von $\mathrm{CO}_{2}$.

Nachdem kürzlich die beiden stereoisomeren Tetrahydroprephensäuren III und IV synthetisch erhalten und ihre sterische Struktur ermittelt werden konnte $^{8,9}$, lag es nahe, natürliche Prephensäure zu hydrieren und das Reaktionsprodukt III oder IV zuzuordnen. Bei der Empfindlichkeit der Prephensäure mußte allerdings besondere Vorsicht am Platz sein. Einmal ist Aromatisierung am Katalysator zu befürchten, außerdem Hydrogenolyse der durch doppelte Allylstellung aktivierten Hydroxylgruppe.

Zur Auffindung geeigneter Reaktionsbedingungen haben wir als Modell die Hydrierung des schon früher ${ }^{10}$ dargestellten 1-Methyl-1-dichlormethyl-cyclohexadien-(2.5) -ols-(4) $\mathrm{V}$ untersucht.
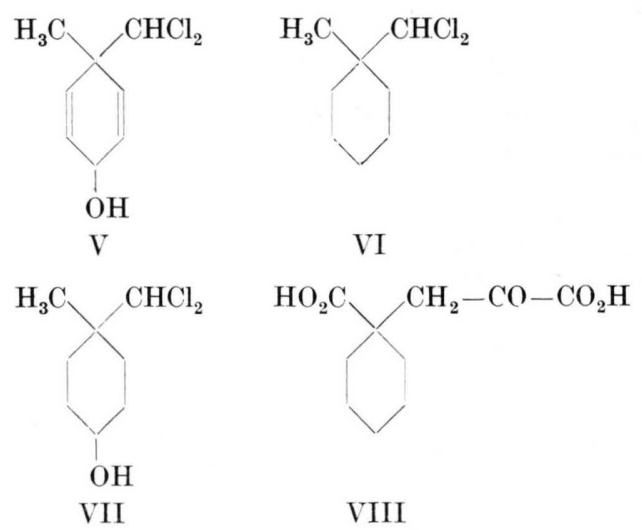

8 H. Plieninger u. H. J. Grasshoff, Chem. Ber. 99, 1973 [1957].

9 H. Plieninger u. G. Keilich, Chem. Ber. 92, 2897 [1959].

10 H. Plieninger u. G. Keilich, Chem. Ber. 91, 1891 [1958].

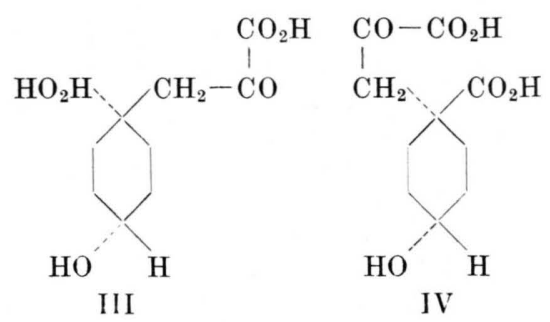

In Gegenwart von Palladium auf Kohle in Methanol werden 3 Moleküle Wasserstoff aufgenommen; unter reduktiver Abspaltung der Hydroxylgruppe entsteht 1-Methyl-1-dichlormethyl-cyclohexan VI. Beim gleichen Versuch mit Palladium auf Bariumsulfat in Methanol mit etwas Triäthylamin wird die für zwei Doppelbindungen berechnete Menge Wasserstoff aufgenommen. Man erhält ohne Hydrogenolyse 1-Methyl-1-dichlormethyl-4-hydroxy-cyclohexan VII in guter Ausbeute. Ohne Hydrogenolyse verlief die Hydrierung mit Raney-Nickel in alkalischer Lösung beim 1-Methyl-1-trichlormethyl-cyclohexadien-(2.5)-ol-(4). In diesem Fall werden auch die Halogenatome durch Wasserstoff ersetzt. Man erhält 4.4-Dimethyl-cyclohexanol.

Danach schien Palladium auf Bariumsulfat ein günstiger Katalysator zu sein.

Aus einer Neurospora-Mutante ${ }^{*}$ haben wir $500 \mathrm{mg}$ Ba-Prephenat gewonnen, das nach der kolorimetrischen Bestimmung (Umwandlung in Phenylbrenztraubensäure und Reaktion mit Eisen(III) -chlorid) etwa 70-proz. war. Bei der Hydrierung mit Palladium/Bariumsulfat in wäßriger Lösung bei $p_{\mathrm{H}} 9,5$ bis 10 werden bei $20{ }^{\circ} \mathrm{C} 55 \mathrm{~cm}^{3}$ Wasserstoff aufgenommen. Das Reaktionsprodukt wird mit salzsaurer 2.4-Dinitrophenylhydrazin-Lösung gefällt und durch Lösen in Alkohol und Fällen mit Wasser gereinigt. Da die Schmelzpunkte und IR-Spektren der Dinitrophenylhydrazone von III und IV nicht charakteristisch sind, wurde eine papierchromatographische Trennung ausgearbeitet. Es wurde absteigend im Durchlaufchromatogramm entwickelt. Mit einem Gemisch Butanol/Eisessig/Wasser/Heptan 20 : $5: 20: 5$ auf Schleicher \& Schüll-Papier Gl 2043 läßt sich zeigen, daß im Hydrierungsprodukt der Prephensäure keine Phenylbrenztraubensäure enthalten ist. Die Ketosäure läßt sich mit diesem Gemisch gut entwickeln, während die Verbindungen

\footnotetext{
* R. L. Metzenberg u. H. K. Mitchell, Arch. Biochem. Biophysics 64, 51 [1956]. Die Kultur haben wir dankenswerterweise von Dr. H. K. Mitchell erhalten.
} 
III und IV sowie das Hydrierungsprodukt der Prephensäure am Startfleck haften bleiben.

Größere Schwierigkeiten macht die Auftrennung von III und IV (immer als Dinitrophenylhydrazone). Mit normalen Laufmitteln und Papieren konnte keine Trennung erzielt werden. Erst nach Imprägnierung des oben genannten Papiers mit 5-proz. Boraxlösung und Trocknen des Papiers an der Luft, lassen sich mit einem Gemisch Butanol/ Äthanol/Wasser 4:1:5 die Stereoisomeren gut trennen. Offenbar bewirkt die Borsäure eine Komplexbindung zwischen der Hydroxylgruppe und dem Dinitrophenylhydrazon-Rest, so daß sich die Isomeren besser unterscheiden *

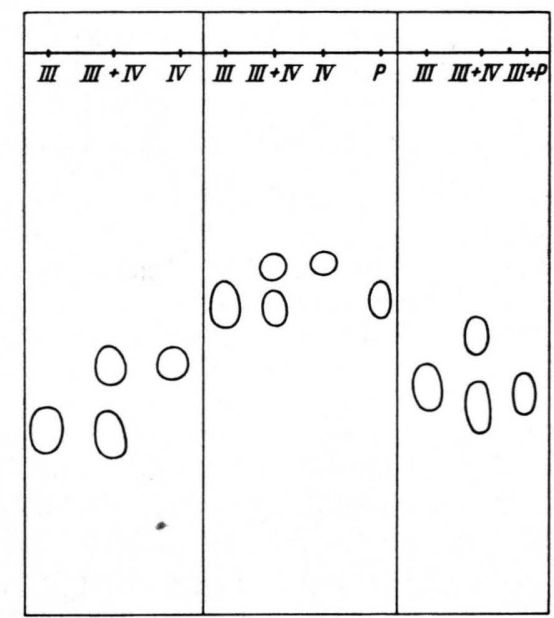

Abb. 1. Papierchromatographischer Vergleich der hydrierten natürlichen Prephensäure P mit den beiden synthetischen isomeren Tetrahydroprephensäuren III und IV (als Dinitrophenylhydrazone).

Die Abb. 1 zeigt die Chromatogramme. Ganz links sieht man die Verbindungen III und IV, dazwischen die Auftrennung eines Gemischs aus III und IV. Das mittlere Chromatogramm zeigt das gleiche, nur daß rechts das Dinitrophenylhydrazon der hydrierten Prephensäure [P] mitläuft. Der Fleck tendiert deutlich nach III. Im rechten Chromatogramm sieht man links reines III; in der Mitte ein aufgetrenntes Gemisch von III und IV und rechts ein Gemisch von III mit P. Hier zeigt sich eindeutige Identität von $\mathrm{P}$ mit III, womit bewiesen ist, daß der Prephensäure die Struktur X zukommt. Außerdem wird durch diese Versuche die bisher angenommene Struktur der Prephensäure eindeutig bewiesen, da

* Den Vorschlag, boratgepuffertes Papier zu verwenden, verdanke ich Herrn Prof. Dr. Th. Wieland. ihr Hydrierungsprodukt mit einer eindeutig synthetisierten Verbindung übereinstimmt.

Schließlich wurde noch die Verbindung VIII synthetisiert und zu den Vergleichen mit herangezogen. Diese Verbindung wäre zu erwarten, wenn bei der Hydrierung der Prephensäure Hydrogenolyse eingetreten wäre. Das Dinitrophenylhydrazon von VIII läuft unter den oben beschriebenen Bedingungen sehr viel schneller als III oder IV und bleibt beim Durchlaufchromatogramm meist nicht auf dem Papier. Bei der Hydrierung des Ba-Prephenats unter den von uns gewählten Bedingungen tritt demnach weder Aromatisierung noch Hydrogenolyse ein.

Die sterischen Befunde stehen in guter Übereinstimmung mit einer Untersuchung von SPRINSON ${ }^{11}$, wonach Prephensäure offenbar aus 3-Enolpyruvylshikimisäure-5-phosphat (IX) nach Art einer para$\mathrm{Cl}$ a is en-Umlagerung entsteht. Der letzte Reaktionsschritt vor der Prephensäure ist demnach der folgende:

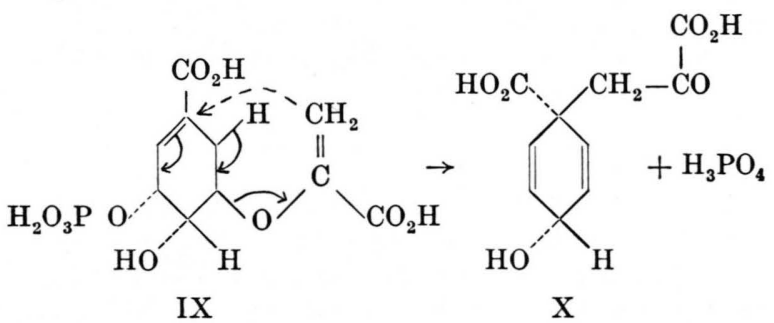

\section{Beschreibung der Versuche}

Hydrierung von 1-Methyl-dichlormethyl-cyclohexadien(2.5)-ol-(4) (V) in Gegenwart von Pd/Kohle zu 1-Methyl-1-dichlormethyl-cyclohexan VI

$1,92 \mathrm{~g}(0,01 \mathrm{Mol}) \mathrm{V}$ werden in $100 \mathrm{~cm}^{3}$ Methanol in Gegenwart von $\mathrm{Pd} /$ Kohle bei Atmosphärendruck und Zimmertemperatur hydriert. Die aufgenommene Wasserstoffmenge entspricht der Hydrierung der Doppelbindungen und Hydrogenolyse der OH-Gruppe $(0,03 \mathrm{Mol})$. Man saugt vom Katalysator ab, destilliert das Lösungsmittel i. Vak. ab und erhält ein helles, fast farbloses Öl, das Chlor enthält. Ausbeute 93,5\% der Theorie.

Das IR-Spektrum zeigt, daß es sich bei dem Öl um ein Cyclohexanderivat ohne Hydroxylgruppe handelt.

Hydrierung von 1-Methyl-1-dichlormethyl-cyclohexadien(2.5)-ol-(4) ( $\mathrm{V}$ ) in Gegenwart von $\mathrm{Pd} / \mathrm{BaSO}_{4} \mathrm{zu}$ 4-Methyl-4-dichlormethylcyclohexanol VII

$1,92 \mathrm{~g}(0,01 \mathrm{Mol}) \mathrm{V}$ werden in $100 \mathrm{~cm}^{3}$ Methanol und $2 \mathrm{~cm}^{3}$ Triäthylamin in Gegenwart von $\mathrm{Pd} / \mathrm{BaSO}_{4}$ bei Atmosphärendruck und Zimmertemperatur unter

11 D. Sprinson, Biochem. Biophys. Res. Communications, im Druck. 
Schütteln hydriert. Die Wasserstoffaufnahme entspricht der für zwei Doppelbindungen berechneten Menge. Nach dem Absaugen des Katalysators wird das Lösungsmittel zuletzt i. Vak. abdestilliert; es bleibt ein öliger Rückstand, der beim Stehen in Eis kristallisiert. Durch Umkristallisieren aus Petroläther erhält man weiße Spieße vom Schmp. $79-80^{\circ}$ C. Der Mischschmelzpunkt mit dem Ausgangsmaterial (Schmp. $82{ }^{\circ} \mathrm{C}$ ) liegt bei $55-60{ }^{\circ} \mathrm{C}$. Ausbeute $90 \%$ der Theorie.

$\mathrm{C}_{8} \mathrm{H}_{14} \mathrm{Cl}_{2} \mathrm{O}(197,1) \quad$ Ber. C 48,80 H 7,16 Cl 36,00. Gef. C 48,96 H 7,41 Cl 35,76.

Hydrierung von 1-Methyl-1-trichlormethyl-cyclohexadien-(2.5)-ol-(4) in Gegenwart von Raney-Nickel zu 4.4-Dimethyl-cyclohexanol

$2,26 \mathrm{~g} \quad(0,01 \mathrm{Mol}) \quad 1$-Methyl-1-trichlormethyl-cyclohexadien-(2.5)-ol-(4) werden in $100 \mathrm{~cm}^{3} 5$-proz. methanolischer Kalilauge in Gegenwart von Raney-Nickel hydriert. Die aufgenommene Wasserstoffmenge entspricht 2 Doppelbindungen und dem Ersatz von 3 ClAtomen durch Wasserstoff. Man saugt vom Katalysator und dem teilweise ausgefallenen $\mathrm{KCl}$ ab und destilliert das Lösungsmittel i. Vak. ab. Der Rückstand wird in Benzol aufgenommen, vom $\mathrm{KCl}$ abfiltriert und die benzolische Lösung getrocknet. Das Benzol wird i. Vak. eingedampft, es bleibt ein helles, chlorfreies Öl, zurück. Ausbeute 1,1 g (61\% d. Th.).

\section{p-Nitrobenzoylester des 1.1-Dimethyl-cyclohexanols-(4)}

$500 \mathrm{mg}$ des oben erhaltenen Öles werden mit $800 \mathrm{mg}$ p-Nitrobenzoylchlorid in $25 \mathrm{~cm}^{3}$ Pyridin (über $\mathrm{KOH}$ getrocknet und destilliert) 15 Min. unter Rückfluß gekocht. Nach dem Abkühlen gießt man das Reaktions gemisch in Eiswasser, äthert den abgeschiedenen Ester aus, wäscht die ätherische Lösung gut mit $2-n$. $\mathrm{NaOH}$, um die entstandene $p$-Nitrobenzoesäure zu entfernen, dann mit verd. Säure und Wasser. Die Ätherlösung wird über wasserfreiem Natriumsulfat getrocknet und der Äther i. Vak. abdestilliert. Der feste, z. T. kristalline Rückstand wird aus Petroläther oder Äthanol umkristallisiert. Schmp. $160-163^{\circ} \mathrm{C}$. Ausbeute 980,5 mg (90\% d. Th.).

$$
\begin{gathered}
\mathrm{C}_{15} \mathrm{H}_{19} \mathrm{O}_{4} \mathrm{~N} \quad \text { (277,3) Ber. N 5,05, Gef. N } 4,97 . \\
\text { [1-Cyan-cyclohexyl-(1)]- } \\
\text { cyanbrenztraubensäureäthylester }
\end{gathered}
$$

$4 \mathrm{~g}(0,1 \mathrm{Mol})$ Kalium werden in einem Gemisch aus $15 \mathrm{~cm}^{3}$ abs. Äthanol und $70 \mathrm{~cm}^{3}$ abs. Äther, zuletzt unter Erwärmen, gelöst. Die erhaltene Lösung wird unter Eiskühlung mit $15 \mathrm{~g}$ Oxalsäurediäthylester versetzt, wobei sich die Mischung gelb färbt. Man läßt das Gemisch 15 Min. bei $20^{\circ}$ stehen und tropft es innerhalb einer Stde. zu einer Lösung von 8,35 g Cyclohexancarbonsäure-(1)-essigsäure-(1)-dinitril in $400 \mathrm{~cm}^{3}$ abs. Benzol bei $40^{\circ}$. Nach $24 \mathrm{Stdn}$. wird der entstandene gelbliche Niederschlag abgesaugt und mit Äther gewaschen. Der Niederschlag wird in $50 \mathrm{~cm}^{3}$ Wasser gelöst und nach dem Filtrieren bei $0^{\circ}$ mit $5 \mathrm{~cm}^{3}$ konz. Salzsäure versetzt, wobei das Enol ölig ausfällt. Es wird in Äther aufgenommen, mit Wasser gewaschen, und der Äther nach dem Trocknen i. Vak. eingedampft. Der erhaltene ölige Rückstand kristallisiert beim Anreiben mit wenig Äther. Durch Umkristallisieren aus Äther/Petroläther erhält man $6 \mathrm{~g}$ einer farblosen Verbindung vom Schmp. $103-105^{\circ}$, die sich bei längerem Stehenlassen zersetzt. Ausbeute $43 \%$ der Theorie.

$$
\begin{aligned}
& \mathrm{C}_{13} \mathrm{H}_{16} \mathrm{O}_{3} \mathrm{~N}_{2} \quad(248,3)
\end{aligned}
$$

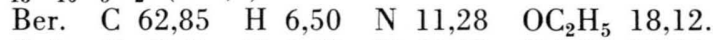

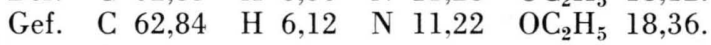

\section{[1-Carboxy-cyclohexyl-(1)]-brenztraubensäure VIII}

$5 \mathrm{~g}$ der im letzten Versuch beschriebenen Verbindung werden mit einer Mischung aus $50 \mathrm{~cm}^{3} 2-n . \mathrm{H}_{2} \mathrm{SO}_{4}$ und $10 \mathrm{~cm}^{3}$ Eisessig $2 \mathrm{Stdn}$. mit Rückfluß gekocht. Nach dem Abkühlen wird dreimal mit Äther extrahiert, der Äther mit $\mathrm{MgSO}_{4}$ getrocknet und eingedampft. Beim Abdampfen des Äthers bleiben $3 \mathrm{~g}$ eines Syrups zurück. Ausbeute $70 \%$ der Theorie.

Mit der wäßrigen Lösung dieser rohen Ketosäure werden einige Derivate hergestellt.

1. Das Dinitrophenylhydrazon. Aus Äthanol/Wasser umkristallisiert: gelbe Nadeln, Schmp. 225-226 ${ }^{\circ}$ Zers. $\mathrm{C}_{16} \mathrm{H}_{18} \mathrm{~N}_{4} \mathrm{O}_{8} \quad(394,3) \quad$ Ber. C 48,75 H 4,59 N 14,19. Gef. C 48,80 H 4,85 N 13,56.

2. Ba-Salz durch Versetzen mit Ba-Acetat-Lösung und Zusatz von Äthanol.

$\mathrm{C}_{10} \mathrm{H}_{12} \mathrm{O} \mathrm{BBa}(349,5) \quad$ Ber. C 34,45 H 3,45 Ba $39,30$. Gef. C 33,76 H 3,94 Ba 38,93 .

Der Diäthylester siedet bei $0,1^{115-18^{\circ}}$.

Die Deutsche Forschungsgemeinschaft sowie der Fonds der Chemischen Industrie hat diese Arbeit großzügig unterstützt. Der B adischen Anilin- und Sod a f a rik haben wir für die Überlassung von Chemikalien zu danken.

Herrn Dr. U. WeIss, Bethesda (USA) danken wir besonders für anregende Diskussionen. 